\title{
Dermoscopic features of congenital hypopigmentary disorders
}

\author{
Subrata Malakar ${ }^{1}$, Harshal Ranglani $^{2}$, Surit Malakar ${ }^{1}$ \\ ${ }^{1}$ Rita Skin Foundation, Kolkata, India, ${ }^{2}$ Skin Secrets, Mumbai, India \\ Corresponding author: Harshal Ranglani, MD, E-mail: drharshalranglani@gmail.com
}

\begin{abstract}
Localized congenital hypopigmentary disorders include conditions like nevus depigmentosus, piebaldism, hypomelanosis of Ito, ash leaf macules and nevus anemicus. These cases are not infrequently seen in clinical practice. However, they often closely mimic each other clinically, and thus pose a diagnostic challenge. In the paediatric age group, it is not always feasible or desirable to do a biopsy. A non-invasive diagnostic tool such as dermoscopy is thus invaluable and obviates the need for any invasive procedures. Differentiation from the far more common disorders such as pityriasis alba and vitiligo is also needed. So, the various salient dermoscopic features of congenital hypopigmentary disorders in order to aid in clinical diagnosis easily are highlighted.
\end{abstract}

Key words: Dermoscopy; Hypopigmentary disorders; Diagnostic dilemmas; Hypopigmentation; Paediatric dermoscopy

\section{INTRODUCTION}

Congenital hypopigmentary disorders are either generalized or localized. They have distinct pathophysiological mechanisms, ranging from defective melanoblast migration to defective melanosome transfer $[1,2]$.

Some of these conditions are important as they may point to the presence of underlying systemic involvement, as in tuberous sclerosis or neurodevelopmental problems associated with hypomelanosis of Ito $[2,3]$. Hence, an accurate diagnosis becomes vital in these cases. However, in the paediatric age group, it is not always feasible or desirable to do a biopsy. A non-invasive diagnostic tool such as dermoscopy is thus invaluable and obviates the need for any invasive procedures $[3,4]$.

In addition, differentiation from the more common, acquired hypopigmentary disorders such as vitiligo and pityriasis versicolor is often needed. Moreover, specific diagnosis of congenital hypopigmentary disorders and confirmation of them being non-vitiliginous would put anxious parents at ease [1].
Hence, a focused assessment and understanding of the dermoscopic features of congenital hypopigmentary disorders is needed, for optimal management of paediatric cases. Here, we highlight the various salient dermoscopic features of congenital hypopigmentary disorders in order to aid in clinical diagnosis easily.

\section{Ash Leaf Macule}

Ash leaf macules (ALM) are characterized by depigmented macules which are lanceolate or ovoid in shape. ALM appears even before the other clinical signs of tuberous sclerosis are evident. Three or more hypopigmented macules of tuberous sclerosis are seen in $97 \%$ of patients at birth or shortly after. Clinically, the lesions appear as hypopigmented, lanceolate or ovoid macules (Fig. la). Sometimes, they pose a diagnostic dilemma, especially in absence of other signs of tuberous sclerosis.

Dermoscopy shows white areas corresponding to areas of complete pigment loss, whereas in other zones, some faint pigmentation may be seen (Fig. lb). The margins are typically irregular and ill-defined, with

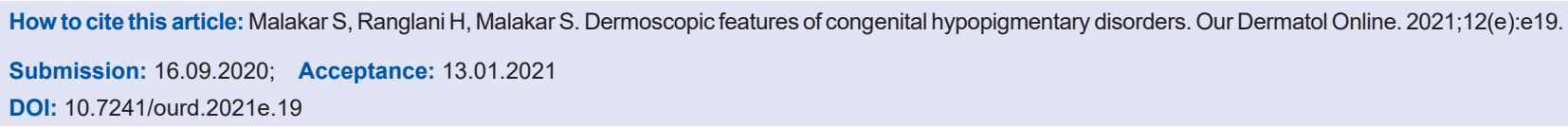


there being no evidence of perifollicular pigmentation or leucotrichia.

ALM is a prime example of how a dermatological sign can lead to the diagnosis of an internal disease, in this case: tuberous sclerosis. While there are dermoscopic findings listed for adenoma sebaceum and periungual fibromas, data on ash leaf macules is sparse.

\section{Nevus Depigmentosus}

Nevus depigmentosus (ND) is a common nevoid hypopigmentary condition characterized by white patches appearing on the skin at birth or may become apparent during infancy or early childhood [3-5]. Clinically, ND shows well-defined hypopigmented patches with serrated borders. It is often confused with vitiligo. It remains stable in shape but increases in size proportionate to the growth of the child.

Dermoscopic findings include faint pigment network present uniformly throughout the lesion, perifollicular pigmentation and pseudopods at periphery representing serrated borders (Fig. 2) [5-7].

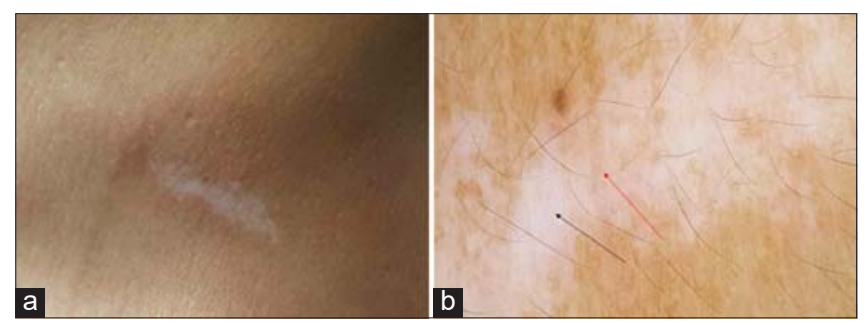

Figure 1: $(a$ and $b)$ Depigmented macule on the anterior part of the neck. Polarized dermoscopy of ash leaf macule shows white areas with zones of total loss pigment network (black arrow) among the zones of faint pigment network (red arrow). [DermLite ${ }^{\mathrm{TM}}$ DL4 (3Gen Inc., San Juan Capistrano, CA, USA), Original magnification $\times 10$ ].

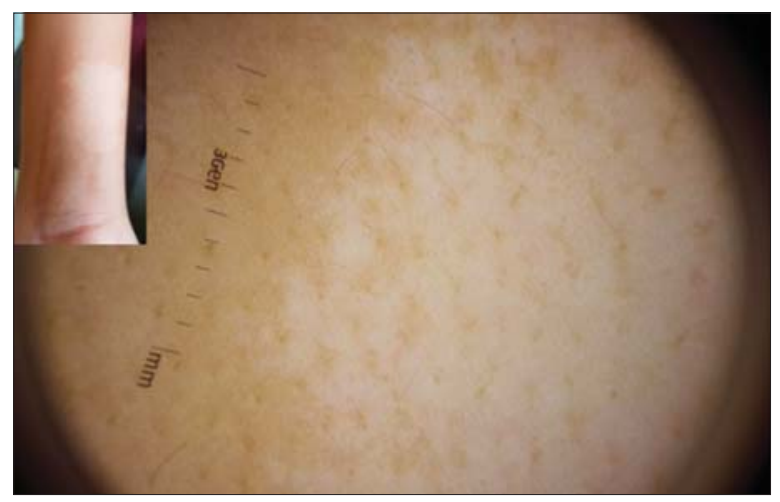

Figure 2: Well defined white patch of nevus depigmentosus with serrated borders on the forearm. Dermoscopy shows subtle pigment network all throughout the patch with pseudopods at the periphery. [DermLite ${ }^{\mathrm{TM}}$ DL4 (3Gen Inc., San Juan Capistrano, CA, USA), Original magnification $\times 10]$.
Peripheral extensions of white areas appear as pseudopods, arising from the border of the lesions (Figs. 3 and 4). Histopathologic studies have shown that the staining ability of Fontana-Masson in lesional skin is less as compared to normal skin. However, the number of melanocytes in lesional and nonlesional skin have been found to be the same. Electron microscopic findings noted are : significant reduction in the number of melanosomes in melanocytes and the presence of some membrane-bound aggregated structures in the keratinocytes. This feature of ND gives affirmation to the fact that melanosomes are not transferred to the keratinocytes even though the number of melanocytes is histologically normal. So, nevus depigmentosus is caused by the functional defects of melanocytes and the morphologic abnormalities of melanosomes [4]. Here, presence of faint or subtle pigmentation in lesion indicates that melanin is present in the epidermis, albeit in lesser quantities.

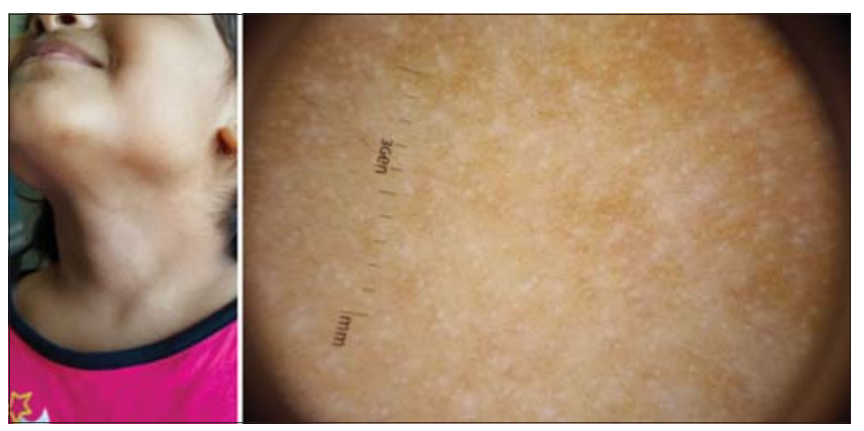

Figure 3: Clinical picture showing segmental nevus depigmentosus over the neck. Dermoscopy reveals faint pigment network and normal black colored hairs. Pinpoint white dots (eccrine or follicular openings) show peripheral pigmentation. [DermLite ${ }^{\mathrm{TM}}$ DL4 (3Gen Inc., San Juan Capistrano, CA, USA), Original magnification $\times 10]$.

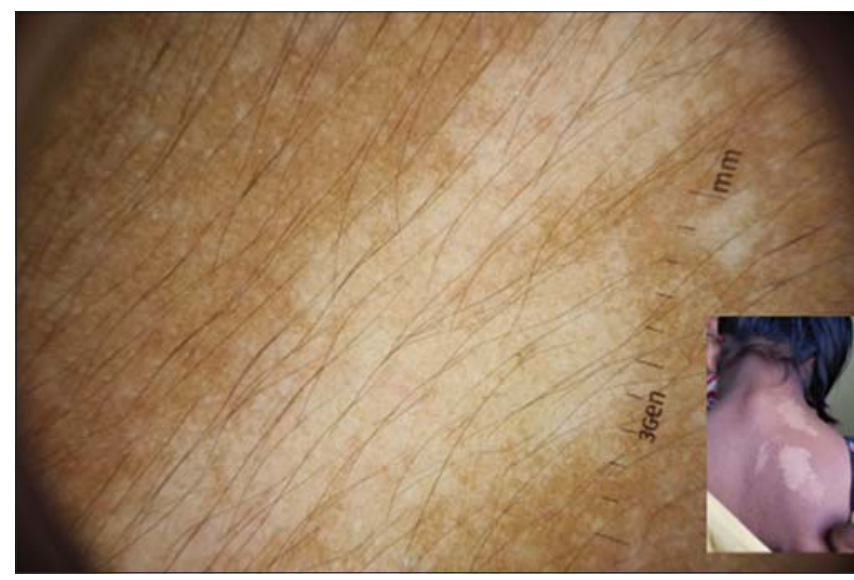

Figure 4: Characteristic dermoscopic features of ND noted here are faint pigment network present uniformly throughout the lesion, along with retention of perifollicular pigment. [DermLite ${ }^{\mathrm{TM}}$ DL4 (3Gen Inc., San Juan Capistrano, CA, USA), Original magnification $\times 10]$. 
Perifollicular pigment is also a prominent feature of ND [5]. The reason for this is hypothesized as increased transfer of melanosomes to hair bulb cells. Leucotrichia is absent in ND. The confusion arises with vitiligo, in which leucotrichia may or may not be seen. Notably, pseudopods are not observed in dermoscopy of vitiligo; instead, perilesional and reverse pigmentation is frequently seen [8].

\section{Hypomelanosis of Ito}

Now included in the group of disorders of 'pigmentary mosaicism', hypomelanosis of Ito is characterized by patterned streaks of hypopigmentation $[9,10]$. The linear streaks and whorls tend to follow lines of mosaicism (lines of Blaschko) or a phylloid pattern of mosaic distribution (Figs. $5 \mathrm{a}$ and $5 \mathrm{~b}$ ). Around $30 \%$ of pediatric patients may have associated abnormalities of the eyes, bones or CNS, but they occur in those with widespread skin involvement [10].

Dermoscopic findings are the presence of a uniform faint pigment network with pigment blotches and an irregular and cloudy border (Figs. 6a and 6b). The significant reduction of melanocytes in hypomelanosis of Ito is reflected in the incomplete, faint reticular pigment pattern, whereas a reduction in melanin results in a blotchy type of pigmentation in the remaining areas.

It closely resembles nevus depigmentosus. The pathophysiological difference between the two conditions is as follows:

- Nevus depigmentosus: transfer defect of melanosomes

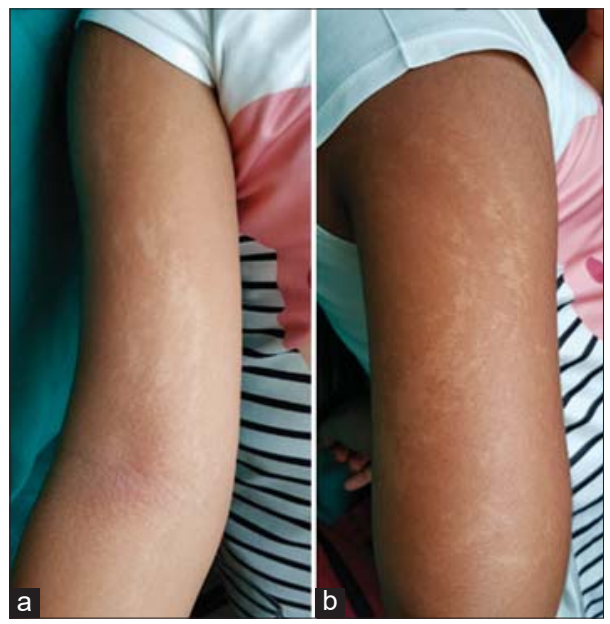

Figure 5: ( $a$ and b) Linear hypopigmented streaks over the arms in a case of hypomelanosis of Ito, seen along the lines of Blaschko.
- Hypomelanosis of Ito: significant reduction of melanosomes and melanocytes in a patchy distribution, with no transfer defect.

\section{Piebaldism}

It is characterized by congenital patterned areas of depigmentation, that may or may not include a white lock of hair above the forehead (white forelock) in affected individuals. It results from defective migration of melanoblasts from the neural crest to the ventral midline and a defect in the differentiation of melanoblasts to melanocytes. So, common sites include the anterior abdomen, anterior aspect of the knees and arms [11].

In piebaldism there are depigmented patches interspersed with islands of normal or increased pigmentation (Figs. 7a and 7b). In contrast to vitiligo, piebaldism presents at birth, with lesions lacking convex borders, and being present mainly on the ventral surfaces.

Key dermoscopic features are the presence of vast areas of depigmentation and a distorted melanocytic network (Fig. 8) [11]. The areas of depigmentation are a result of defective migration of melanoblasts from the neural crest to the ventral midline. The distorted melanocytic network with reduced intensity of pigmentation results from a defect in the differentiation of melanoblasts to melanocytes.

There is absence of perifollicular pigment. We hypothesize that the absence of perifollicular pigment may be due to defect in transfer of melanosomes from the melanocytes in the hair bud to the surrounding

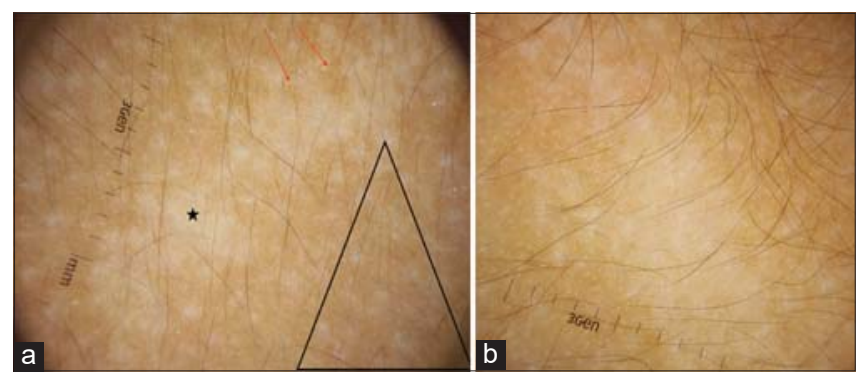

Figure 6: (a) Dermoscopic features of hypomelanosis of Ito include a diffuse light brown background (black star). In addition, there is also presence of blotchy pigmentation (red arrows). Irregular \& cloudy border is a distinguishing feature here (black triangle). [DermLite ${ }^{\mathrm{TM}}$ DL4 (3Gen Inc., San Juan Capistrano, CA, USA), Original magnification $\times 10$ ]. (b) Hypomelanosis of Ito: Presence of blotchy pigmentation along with a cloudy border can be noted. [DermLite ${ }^{\mathrm{TM}} \mathrm{DL} 4$ (3Gen Inc., San Juan Capistrano, CA, USA), Original magnification × 10]. 
Table 1: Key dermoscopic features of various congenital hypopigmentary disorders

\begin{tabular}{|c|c|c|c|c|}
\hline Features & Nevus depigmentosus & Hypomelanosis of Ito & Ash leaf macule & Piebaldism \\
\hline Background color & Light brown & Light brown & Light brown and white & White \\
\hline Pigment network & $\begin{array}{l}\text { Uniformly faint pigment network } \\
\text { throughout the lesion }\end{array}$ & $\begin{array}{l}\text { Uniform faint reticular pigment } \\
\text { network with pigment blotches }\end{array}$ & $\begin{array}{l}\text { Zones of faint pigment network with } \\
\text { zones of total loss of pigment network }\end{array}$ & $\begin{array}{l}\text { Distorted pigment network } \\
\text { with absence at places }\end{array}$ \\
\hline $\begin{array}{l}\text { Perifollicular } \\
\text { pigmentation }\end{array}$ & Mostly present & Absent & Absent & Absent \\
\hline $\begin{array}{l}\text { Islands of normal } \\
\text { pigment network }\end{array}$ & Absent & Absent & Absent & Present \\
\hline Margin of the patch & $\begin{array}{l}\text { Serrated(accentuated) or wavy } \\
\text { or pseudopod like extension }\end{array}$ & Irregular and cloudy & III-defined and irregular & Irregular \\
\hline Leucotrichia & Absent & Absent & Absent & $+/-$ \\
\hline
\end{tabular}

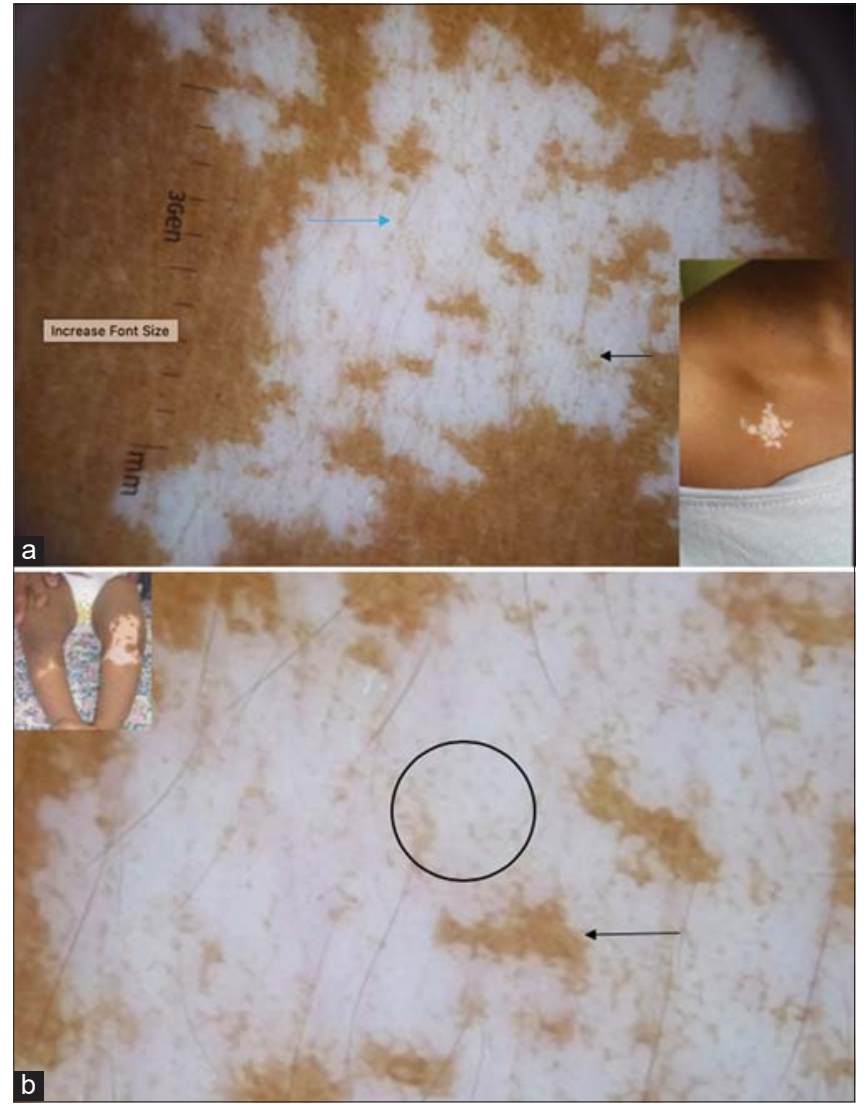

Figure 7: (a) Polarized dermoscopy of piebaldism reveals distorted pigment network (black arrow), absence of perifollicular pigment (blue arrow) and vast areas of depigmentation. (Inset shows the clinical picture: depigmented patch with irregular margins, present over the anterior chest). [DermLite ${ }^{\mathrm{TM}}$ DL4 (3Gen Inc., San Juan Capistrano, CA, USA), Original magnification $\times 10$ ] (b) Magnified dermoscopic image showing areas of distorted pigment network (black circle) and islands of normal pigmentation (black arrow), on a white background. (Inset shows patches of piebaldism present over both the knees. Note the depigmented patches with interspersed islands of pigmentation.) [DermLite ${ }^{\mathrm{TM}}$ DL4 (3Gen Inc., San Juan Capistrano, CA, USA), Original magnification $\times 10]$.

keratinocytes. However, further studies may be necessary to substantiate this. Islands of normal or hyperpigmented skin may be seen. Irregular margins are present. Leucotrichia may or may not be present. It is frequently seen with poliosis, as expected. However,

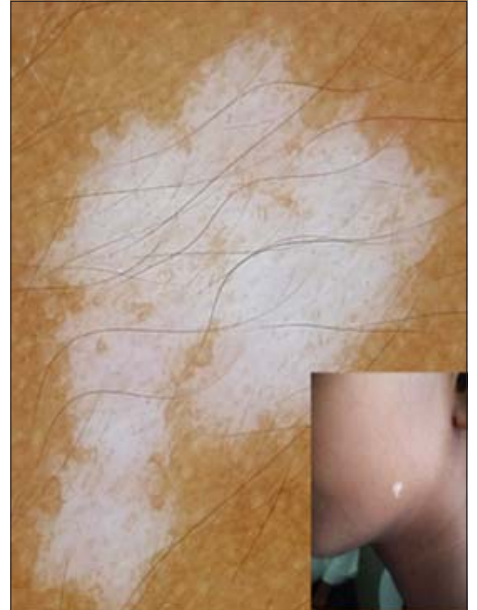

Figure 8: Tiny, hypopigmented patch over the left mandibular area, mimicking vitiligo. However, dermoscopic examination reveals the most striking feature of piebaldism: distorted pigment network. [DermLite ${ }^{\top M} \mathrm{DL} 4$ (3Gen Inc., San Juan Capistrano, CA, USA), Original magnification × 10].

presence of leucotrichia at sites of piebaldism other than the forehead needs to be studied more. In our experience, we have noted a lack of leucotrichia in the patches of piebaldism noted on the trunk.

\section{Nevus Anemicus}

The color of the skin is determined by melanin as well as hemoglobin in the vessels. Nevus anemicus is a hypopigmented lesion, characterized by welldefined, irregularly shaped, confluent macules. On histopathological examination, the number of melanocytes and melanin content in the skin is normal. The underlying mechanism is an aberrant, hypersensitive response to catecholamines, resulting in pallor of the skin. Hence, it has been called a pharmacological naevus. When the skin in these lesions is rubbed, no red coloring is observed as there is no vasodilatation possible. Since diascopy gives a fairly certain diagnosis of nevus anemicus, dermoscopy is not needed. Still, it is an important differential for congenitally present hypopigmented macules [7]. 


\section{CONCLUSION}

Thus, by a systematic assessment of various parameters listed below, a diagnosis of various congenital hypopigmentary disorders can be made easily (Table 1).

- Border of the lesion

- Pigment network in the lesion: accentuated/ attenuated/normal

- Perifollicular pigmentation / accentuation

- Leukotrichia

It does require training the eye to be able to delineate these features.

It is thus important to know the different kinds of congenital hypopigmentary disorders and their salient dermoscopic features as it can greatly aid their diagnosis in the paediatric population, which commonly presents with these. Thus, dermoscopy can once again come to the aid and obviate the need for a biopsy in particularly difficult patients.

\section{REFERENCES}

1. Deepadarshan K, Gangadhar B, Mallikarjun M. Cutaneous hypopigmentary disorders - An observational study. Our Dermatol Online. 2016;7:145-8.
2. van Geel N, Speeckaert M, Chevolet I, De Schepper S, Lapeere H, Boone B, Speeckaert R. Hypomelanoses in children. J Cutan Aesthet Surg. 2013;6:65-72.

3. Gajjar PC, Mehta HH, Gosai M. Dermoscopy of congenital dermatoses in pediatric age group: an Observational study. Indian J Paediatr Dermatol. 2019;20:219-26.

4. Al-Refu K. Dermoscopy is a new diagnostic tool in diagnosis of common hypopigmented macular disease: A descriptive study. Dermatol Reports. 2018;11:7916.

5. Deb S, Sarkar R, Samanta AB. A brief review of nevus depigmentosus. Pigment Int. 2014;1:56-8.

6. Malakar S, Mukherjee SS, Malakar S. Uniform faint reticulate pigment network - A dermoscopic hallmark of nevus depigmentosus. Our Dermatol Online. 2018;9:225-6.

7. Malakar S, Mukherjee S. Differentiation of nevus depigmentosus, ash leaf macule and nevus anemicus. In: Chatterjee M, Neema S, Malakar S, editors. Dermoscopy in Darker Skin. 1st ed. New Delhi: Jaypee; 2017. p. 39-41.

8. Thatte SS, Khopkar US. The utility of dermoscopy in the diagnosis of evolving lesions of vitiligo. Indian J Dermatol Venereol Leprol. 2014;80:505-8.

9. Abid Keen M. Hypomelanosis of Ito: Report of two cases. Our Dermatol Online. 2015;6:433-5.

10. Kumar HY, Jayaprasad S. Hypomelanosis of Ito: A rare cutaneous syndrome. Int J Health Allied Sci. 2013;2:203-5.

11. Malakar S, Mukherjee SS, Malakar S. Looking through the dermoscope in a case of piebaldism. Our Dermatol Online. 2019;10:312-3

Copyright by Harshal Ranglani. This is an open-access article distributed under the terms of the Creative Commons Attribution License, which permits unrestricted use, distribution, and reproduction in any medium, provided the original author and source are credited.

Source of Support: Nil, Conflict of Interest: None declared. 